\title{
Suppression of high transverse momentum hadrons at RHIC by (pre-) hadronic final state interactions
}

\author{
W. Cassing ${ }^{a}$, K. Gallmeister ${ }^{a, *}$ C. Greiner ${ }^{b}$ \\ ${ }^{a}$ Institut für Theoretische Physik, Universität Giessen, Heinrich-Buff-Ring 16, \\ D-35392 Giessen, Germany \\ ${ }^{\mathrm{b}}$ Institut für Theoretische Physik, Universität Frankfurt, Robert-Mayer-Str. 8-10, \\ D-60054 Frankfurt, Germany
}

\begin{abstract}
We investigate transverse hadron spectra from proton+proton, deuteron $+\mathrm{Au}$ and $\mathrm{Au}+\mathrm{Au}$ collisions at $\sqrt{s}=200 \mathrm{GeV}$ and $\sqrt{s}=17.3 \mathrm{GeV}$ within the Hadron-StringDynamics (HSD) approach which is based on quark, diquark, string and hadronic degrees of freedom as well as PYTHIA calculations for the high $p_{\perp}$ spectra. The comparison to experimental data on transverse mass spectra from $\mathrm{p}+\mathrm{p}, \mathrm{d}+\mathrm{Au}$ and $\mathrm{Au}+\mathrm{Au}$ reactions shows that pre-hadronic effects are responsible for both the hardening of the spectra for low transverse momenta (Cronin effect) as well as the suppression of high $p_{\perp}$ hadrons. The interactions of formed hadrons are found to be negligible in central $\mathrm{Au}+\mathrm{Au}$ collisions at $\sqrt{s}=200 \mathrm{GeV}$ for $p_{\perp} \geq 6 \mathrm{GeV} / \mathrm{c}$, but have some importance for the shape of the ratio $R_{A A}$ at lower $p_{\perp}$ values $(\leq 6 \mathrm{GeV} / \mathrm{c})$. The large suppression seen experimentally is attributed to the inelastic interactions of 'leading' pre-hadrons with the dense environment, which could be partly of colored partonic nature in order to explain the large attenuation seen in most central $\mathrm{Au}+\mathrm{Au}$ collisions.
\end{abstract}

Key words: Relativistic heavy-ion collisions, Meson production, Quark-gluon plasma, Fragmentation into hadrons

PACS: 25.75.-q, 13.60.Le, 12.38.Mh, 13.87.Fh

*

Email address: Kai.Gallmeister@theo.physik.uni-giessen.de

(K. Gallmeister).

Preprint submitted to Elsevier Science

6 July 2018 


\section{Introduction}

The phase transition from partonic degrees of freedom (quarks and gluons) to interacting hadrons - as expected in the early universe shortly after the "big bang" - is a central topic of modern high-energy physics. In order to understand the dynamics and relevant scales of this transition laboratory experiments under controlled conditions are presently performed with ultrarelativistic nucleus-nucleus collisions. Hadronic spectra and relative hadron abundancies from these experiments reflect important aspects of the dynamics in the hot and dense zone formed in the early phase of the reaction. In fact, estimates based on the Bjorken formula [1] for the energy density achieved in central $\mathrm{Au}+\mathrm{Au}$ collisions suggest that the critical energy density for the formation of a quark-gluon plasma (QGP) of 0.7 to $1 \mathrm{GeV} / \mathrm{fm}^{3}$ [2] is by far exceeded in the initial phase for a couple of $\mathrm{fm} / \mathrm{c}$ at Relativistic Heavy Ion Collider (RHIC) energies [3], but it is still the aim to unambiguously identify the formation and properties of this new phase.

Presently, transverse momentum (or transverse mass) spectra of hadrons are in the center of interest. Here the suppression of high transverse momentum hadrons is investigated in $\mathrm{Au}+\mathrm{Au}$ reactions relative to $\mathrm{p}+\mathrm{p}$ collisions at $\mathrm{RHIC}$ energies of $\sqrt{s}=200 \mathrm{GeV}$ [4], since the propagation of a fast quark through a colored medium (QGP) is expected to be different from that in cold nuclear matter as well as in the QCD vacuum due to the energy loss of induced gluon radiation $[5,6,7,8,9]$. In fact, the PHENIX [10], STAR [11] and BRAHMS [12] collaborations have reported a large relative suppression of hadron spectra for transverse momenta above $p_{\perp} \simeq 3 \cdots 4 \mathrm{GeV} / \mathrm{c}$ which might point toward the creation of a QGP, since this suppression is not observed in $\mathrm{d}+\mathrm{Au}$ interactions at the same bombarding energy per nucleon $[12,13,14]$. Accordingly, the experimental observations are qualitatively in line with expectations from perturbative Quantum Chromo Dynamics (pQCD) [15,16,17,18,19], but do not support the idea of initial state gluon saturation [20]. But, at present, it cannot be ruled out that a larger fraction of the suppression seen in central $\mathrm{Au}+\mathrm{Au}$ collisions might be also due to hadronic final state interactions as suggested in Ref. [21]. It is the aim of this work to quantify the amount of hadronic final state interactions in $\mathrm{d}+\mathrm{Au}$ and $\mathrm{Au}+\mathrm{Au}$ collisions at RHIC energies.

We employ the HSD transport model $[22,23,24]$ for our study. This approach takes into account the formation and multiple rescattering of formed hadrons as well as unformed 'leading' pre-hadrons and thus superseeds the incoherent summation of individual $\mathrm{p}+\mathrm{p}$ collisions. Such transport calculations allow to study systematically the change in the dynamics from elementary baryonbaryon or meson-baryon collisions to proton-nucleus reactions or from peripheral to central nucleus-nucleus collisions in a unique way without changing any parameter. In the HSD approach nucleons, $\Delta$ 's, $\mathrm{N}^{*}(1440), \mathrm{N}^{*}(1535), \Lambda, \Sigma$ and 
$\Sigma^{*}$ hyperons, $\Xi$ 's, $\Xi^{*}$ 's and $\Omega$ 's as well as their antiparticles are included on the baryonic side whereas the $0^{-}$and $1^{-}$octet states are included in the mesonic sector. Inelastic hadron-hadron collisions with energies above $\sqrt{s} \simeq 2.6 \mathrm{GeV}$ are described by the Fritiof model [25] (including Pythia v5.5 with JETSET v7.3 for the production and fragmentation of jets [26]) whereas low energy hadron-hadron collisions are modeled in line with experimental cross sections. We mention that no explicit parton cascading is involved in our transport calculations contrary to e.g. the AMPT model [27].

A systematic analysis of HSD results and experimental data for central nucleusnucleus collisions for $2 \cdots 160 \mathrm{AGeV}$ has shown that the spectra for the 'longitudinal' rapidity distribution of protons, pions, kaons, antikaons and hyperons are in reasonable agreement with available data. Only the pion rapidity spectra are slightly overestimated from AGS to SPS energies [28] which implies, that the maximum in the $K^{+} / \pi^{+}$ratio at $20 \cdots 30 \mathrm{AGeV}$ - seen in central $\mathrm{Au}+\mathrm{Au}$ $(\mathrm{Pb}+\mathrm{Pb})$ collisions [29] - is missed. For a comparison of HSD calculations with experimental data at RHIC energies we refer the reader to Ref. [30].

In this work we concentrate on the transverse momentum dynamics and especially on the very high momentum tail of the hadron spectra. In order to describe these high $p_{\perp}$ spectra, we use the PYTHIA v6.2 event generator [31] for nucleon-nucleon collisions, which describes the high transverse momentum spectra of peripheral nucleus-nucleus collisions from RHIC at $\sqrt{s}=130 \mathrm{GeV}$ very well [21]. We recall, that within this framework also experimental dilepton and direct photon spectra at SPS energies [32] or single lepton spectra at RHIC energies from open heavy quark production [33] are well reproduced.

\section{Transport calculations versus experimental data}

We start with hadron production in $\mathrm{p}+\mathrm{p}$ collisions at the invariant energy $\sqrt{s}=200 \mathrm{GeV}$. The comparison of the calculations for the transverse momentum spectra of charged hadrons from $\mathrm{p}+\mathrm{p}$ collisions at midrapidity with the experimental data of the PHENIX and STAR collaborations [34,11] is shown in Fig. 1, which demonstrates that the description of hadron production in the elementary reaction is quite well under control (see also Ref. [21]).

\subsection{Preliminaries}

Before coming to proton+nucleus or deuteron+nucleus collisions it is important to specify the concept of "leading" (pre-)hadrons and "non-leading" or "secondary" hadrons since this separation is of central importance for the re- 


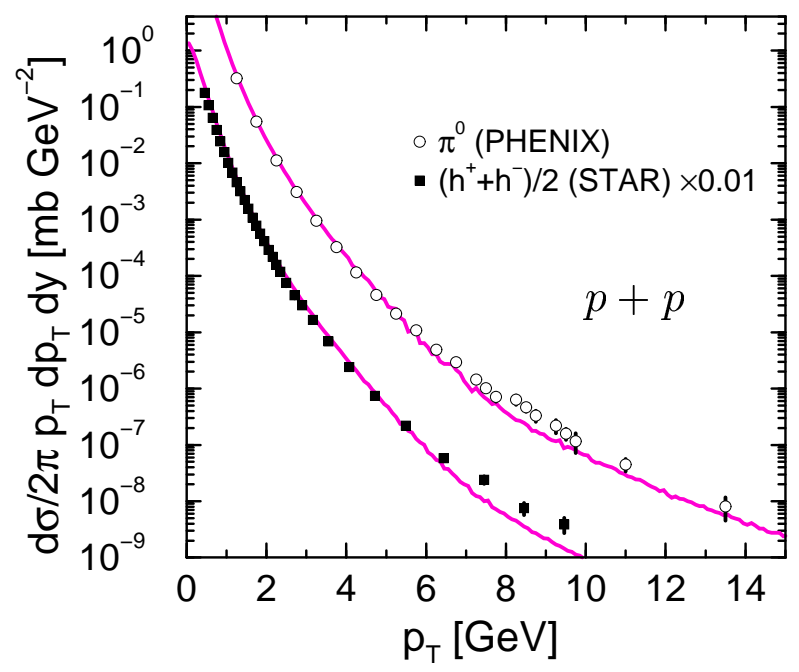

Fig. 1. The invariant cross sections for the production of neutral pions and charged hadrons in $\mathrm{p}+\mathrm{p}$ collisions $(\sqrt{s}=200 \mathrm{GeV})$ at midrapidity as a function of transverse momentum. The Pythia (version v6.2) calculations (solid lines) are compared to the experimental data from RHIC (open circles: PHENIX [34], filled squares: STAR [11]). The charged hadron spectra are divided by a factor of 100 for clarity.

sults to be discussed below. To this aim we recall that in the string picture - in a high energy nucleon+nucleon collision - two (or more) color-neutral strings are assumed to be formed, which phenomenologically describe the low energy "coherent" gluon dynamics by means of a color electric field, which is stretched between the 'colored' ends of each single string (cf. Fig. 2a for an illustration). The latter string ends are defined by the space-time coordinates of the constituents, i.e. a diquark and quark for a "baryonic" string or quark and antiquark for a "mesonic" string. These constituent quarks, diquarks or antiquarks are denoted as "leading" quarks. In line with Ref. [35] these constituent 'partons' are assumed to pick up (almost instantly) an anti-colored parton from the vacuum and achieve color neutrality. These color-neutral objects containing the string ends - are denoted as "leading pre-hadron" (cf. Fig. 2b for an illustration of the string fragmentation in space and time in the rest frame of the string.)

In principle, a leading pre-meson or pre-baryon is able to hold up to 2 (3) leading quarks, but in the case of interest, i.e. midrapidity transverse momentum spectra in heavy-ion collision at $\sqrt{s}=200 \mathrm{GeV}$, the majority of leading mesons/hadrons consists only of one leading quark and some secondary (di-) quarks, as will be discussed below. We note, that this changes dramatically for rapidity regions closer to those of the projectile or target or different $\sqrt{s}$ values, as discussed below.

The time that is needed from the instant of the 'hard' pQCD collision $t_{0}=0$ to the formation of $q \bar{q}$ or $q q \overline{q q}$ pairs from the vacuum $\left(t_{1}>t_{0}\right)$ and for the hadronization of the fragments $\left(t_{2}=\tau_{f}\right)$ we denote as formation time $\tau_{f}$ in line 
with the convention in transport models. For simplicity we assume (in HSD and also for the high $p_{\perp}$ partons), that the formation time is a constant $\tau_{f}$ in the rest frame of each hadron and that it does not depend on the particle species. Other estimates (cf. [21,36,37]) show a more complicated structure and particle dependence (especially for the light pion), but this issue will not be discussed in detail here; nevertheless, we will show results for different formation times and alternative scenarios below. In principle one expects also a distribution in the formation times, however, we here address only the mean value of such an 'unknown' distribution. We recall, that due to time dilatation the formation time $t_{f}$ in any reference frame is then proportional to the energy of the particle

$$
t_{f}=\gamma \cdot \tau_{f}=\frac{E_{h}}{m_{h}} \cdot \tau_{f}
$$

The size of $\tau_{f}$ can roughly be estimated by the time that the constituents of the hadrons (with velocity $c$ ) need to travel a transversal distance of a typical hadronic radius $(0.5 \cdots 0.8 \mathrm{fm})$. Furthermore, since after the formation of a color neutral pre-hadron $\left(t \geq t_{1}\right)$ the Fock-components of this object are widespread in mass according to the uncertainty relation in energy and time, it will take a time of $\Delta E^{-1}$ to form a specific hadronic state of well defined radial excitation, where $\Delta E$ denotes the level distance of the hadronic spectrum.

We assume in our transport simulations that hadrons, whose constituent quarks and antiquarks are created from the vacuum in the string fragmentation (at times $t_{1}$ ), do not interact with the surrounding nuclear medium within their formation time (cf. Fig. 2c for an illustration). For the leading pre-hadrons, i.e. those involving (anti-)quarks or diquarks from the struck nucleons, we adopt a reduced effective cross section $\sigma_{\text {lead }}$ during the formation time $\tau_{f}$ and the full hadronic cross section later on. Since this rough approximation is subject of current debate [35] we will also present alternative models for the cross section of the leading pre-hadrons (see below). The 'default' concept is illustrated in more detail in Fig. 2c where a 'star' signals a possible collision while crossing lines imply that no collision might occur.

Due to time dilatation light particles emerging from the middle of the string can escape the formed 'fireball' without further interaction, if they carry a high momentum relative to the rest frame of the fireball. We note that these arguments are strictly valid only in case of a constant formation time $\tau_{f}$ in the rest frame as described above and implemented here. However, hadrons with transverse momenta larger than $6 \mathrm{GeV} / \mathrm{c}$ predominantly stem from the string ends and therefore can interact directly with a reduced cross section (see below). 
a)

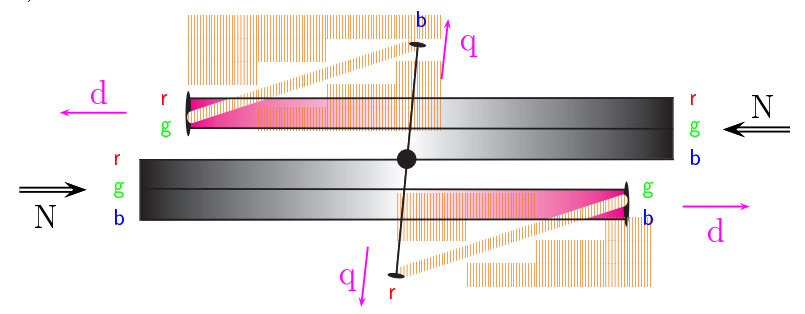

b)

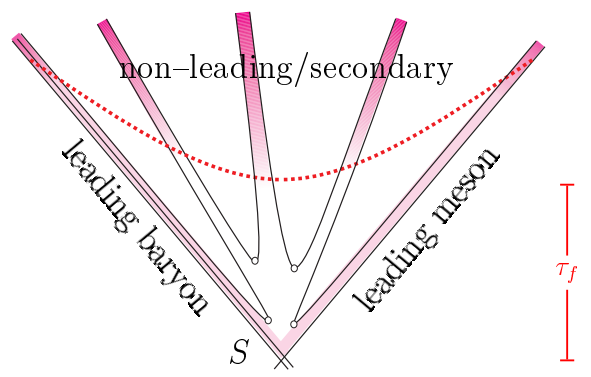

c)

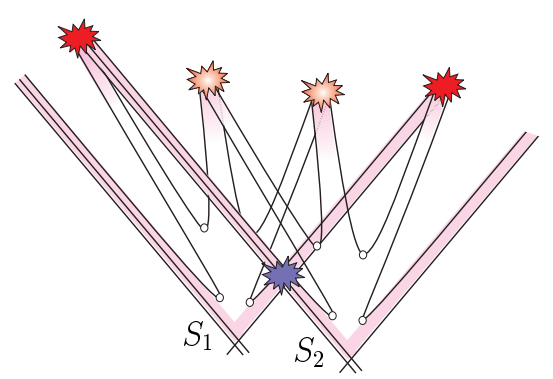

Fig. 2. Upper Part: Illustration of the formation of two strings in a $\mathrm{N}+\mathrm{N}$ collision. The formed strings are color-neutral in total, however, carry (anti-)color at the string-ends, which here are displayed as diquark $(d)$ - quark $(q)$ pairs. The hatched areas denote nonperturbatively interacting partons. Middle Part: The space-time evolution of a string $S$ in its rest frame. Whereas the 'leading' (di-)quarks (represented by the outer lines) form 'leading' pre-hadrons almost instantly, secondary $q \bar{q}$ pairs are excited in the non-perturbative vacuum (after some time delay $t_{1}$ ) and recombine to secondary hadrons (shadowed double lines). The time from the initial creation of the string to the final formation of secondary hadrons is denoted as $\tau_{f}$. The distribution in the formation time is presently unknown and for convenience characterized by a single formation time $\tau_{f}$ for all hadrons (dotted hyperbola). Lower Part: Whereas 'secondary hadrons' are not allowed to scatter during their formation time, they interact with the full hadronic cross section after being formed. The 'leading' color neutral pre-hadrons, however, carrying a constituent quark from the struck hadron, may scatter (in-)elastically with other leading pre-hadrons as well as formed secondary hadrons with a reduced cross section. In the present realization of HSD this reduced cross section is determined in line with the constituent quark model (see text). 


\subsection{Numerical implementation}

For the production and propagation of hadrons with high transverse momentum $(>1.5 \mathrm{GeV} / \mathrm{c})$ we employ a perturbative scheme as also used in Refs. $[38,39,40,41]$ for the charm and open charm degrees of freedom. Each high $p_{\perp}$ hadron is produced in the transport calculation with a weight $W_{i}$ given by the ratio of the actual production cross section divided by the inelastic nucleon-nucleon cross section, e.g.

$$
W_{i}=\frac{\sigma_{N N \rightarrow h\left(p_{\perp}\right)+x}(\sqrt{s})}{\sigma_{N N}^{\text {inelas. }}(\sqrt{s})} .
$$

In the transport simulation we follow the motion of the high $p_{\perp}$ hadrons within the full background of strings/hadrons by propagating them as free particles, i.e. neglecting in-medium potentials, but compute their collisional history with baryons and mesons or quarks and diquarks. For reactions with diquarks we use the corresponding reaction cross section with baryons multiplied by a factor of $2 / 3$. For collisions with quarks (antiquarks) we adopt half of the cross section for collisions with mesons and for the leading pre-hadron (formed) baryon collision a factor of $1 / 3$ is assumed. The elastic and inelastic interactions with their fractional cross section are modeled in the HSD approach in the same way as for ordinary hadrons with the same quantum numbers via the Fritiof model [25] (including Pythia v5.5 with Jetset v7.3 for the production and fragmentation of jets [26]).

The relative quark counting factors mentioned above might appear arbitrary and simplistic. However, this concept - oriented along the additive quark model - has been proven to work rather well for nucleus-nucleus collisions from AGS to RHIC energies $[28,30,42]$ as well as in hadron formation and attenuation in deep inelastic lepton scattering off nuclei [43]. Especially the latter reactions are important to understand the attenuation of pre-hadrons or ordinary hadrons with high momentum in cold nuclear matter [35]. Our studies in Ref. [43] have demonstrated that the dominant final state interactions (FSI) of the hadrons with maximum momentum, as measured by the HERMES collaboration [44], are compatible to the concepts described above. This also holds for antiproton production and attenuation in proton-nucleus collisions at AGS energies [45]. Both independent studies point towards a hadron formation time $\tau_{f}$ in the order of $0.4 \cdots 0.8 \mathrm{fm} / \mathrm{c}$.

As mentioned above, a crucial question for the interaction dynamics is the fraction of leading pre-hadrons to secondary hadrons (or all produced hadrons) as a function of $p_{\perp}$ for the different hadron species. This information is directly extracted from the PYTHIA calculations for different hadrons by explictly tracing back the string fragmentation histories with all their details, i.e., begin- 
ning with the incoming hadrons, following the hard interaction and linking the quark content of the final hadrons back to the partons at the initial string ends. The resulting fraction of leading pre-hadrons to all produced hadrons is displayed in Fig. 3 for $\mathrm{N}+\mathrm{N}$ collisions at $\sqrt{s}=200 \mathrm{GeV}$. We have checked,

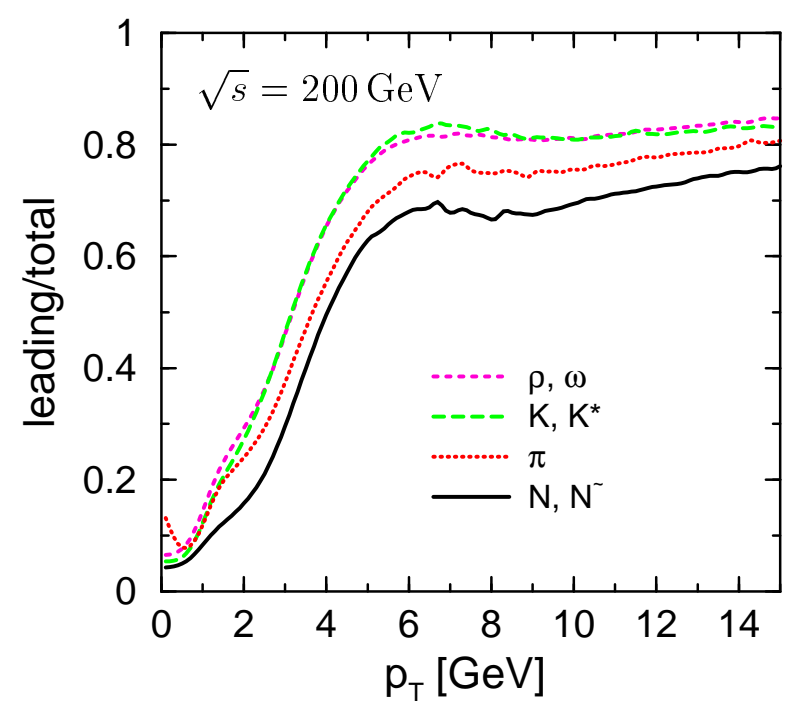

Fig. 3. The ratio of 'leading particles' to 'all produced particles' (before particle decays) in $\mathrm{N}+\mathrm{N}$ collisions $(\sqrt{s}=200 \mathrm{GeV})$ at midrapidity for different particle classes as a function of transverse momentum within the PYTHIA description. (An isospin average in line with central $\mathrm{Au}+\mathrm{Au}$ collisions has been applied.) For the region $p_{\perp}=5 \cdots 7 \mathrm{GeV} / \mathrm{c}$ the theoretical error is of the order \pm 0.05 and to be neglected outside this region.

that in Fig. 3 contributions with 2 (or more) leading particles are negligible and thus the above mentioned "leading" cross sections $\sigma_{\text {lead }}$ as a fraction of the hadronic cross section of $1 / 2$ for mesons and $1 / 3$ for baryons are sustained by simple quark counting rules.

In detail, one notices slight differences between pions and especially antibaryons (not shown), however, the fraction of leading pre-hadrons increases almost linearly with $p_{\perp}$ and approximately saturates above $p_{\perp} \simeq 6 \mathrm{GeV} / \mathrm{c}$ for $\sqrt{s}=$ $200 \mathrm{GeV}$. Thus at high momenta the major fraction of 'hadrons' is of "leading" origin, i.e. pre-hadronic states containing quarks, antiquarks or diquarks from the primary struck nucleons, and may interact according to the assumptions stated above without delay with the (pre-) hadronic environment (cf. Fig. 2c).

\subsection{Initial state Cronin enhancement}

As known from the experimental studies of Refs. $[46,47]$ an enhancement of the high transverse momentum particle cross section from proton-nucleus collisions - relative to scaled $\mathrm{p}+\mathrm{p}$ collisions - is already observed at SPS and 
ISR energies. This 'Cronin effect' is presently not fully understood in its details, but probably related to an increase of the average transverse momentum squared $\left\langle k_{\perp}^{2}\right\rangle$ of the partons in the nuclear medium. One may speculate that such an enhancement of $\left\langle k_{\perp}^{2}\right\rangle$ is due to induced initial semi-hard gluon radiation in the medium, which is not present in the vacuum due to the constraint of color neutrality. A related interpretation has been given by e.g. Kopeliovich et al. $[17,35]$. Since the microscopic mechanisms are beyond the scope of our present analysis, we do not want to comment this any further and simulate this effect in the transport approach by increasing the average $\left\langle k_{\perp}^{2}\right\rangle$ in the string fragmentation with the number of previous collisions $N_{\text {prev }}$ as

$$
\left\langle k_{\perp}^{2}\right\rangle=\left\langle k_{\perp}^{2}\right\rangle_{p p}\left(1+\alpha N_{\text {prev }}\right)
$$

The parameter $\alpha \approx 0.25-0.4$ in (3) is fixed in comparison to the experimental data for $\mathrm{d}+\mathrm{Au}$ collisions $[13,14]$ (see below). The assumption (3) is in line with an independent suggestion in Ref. [48].

In order to show the effect of enhancing the average $\left\langle k_{\perp}^{2}\right\rangle$ in the fragmentation of the string we show in Fig. 4 the ratio of the charged hadron spectra from $p p$ collisions for different $\left\langle k_{\perp}^{2}\right\rangle$ to the spectra for the default value of $\left\langle k_{\perp}^{2}\right\rangle_{p p}=$ $0.36 \mathrm{GeV}^{2}$. These ratios may be denoted as 'Cronin enhancement factors',

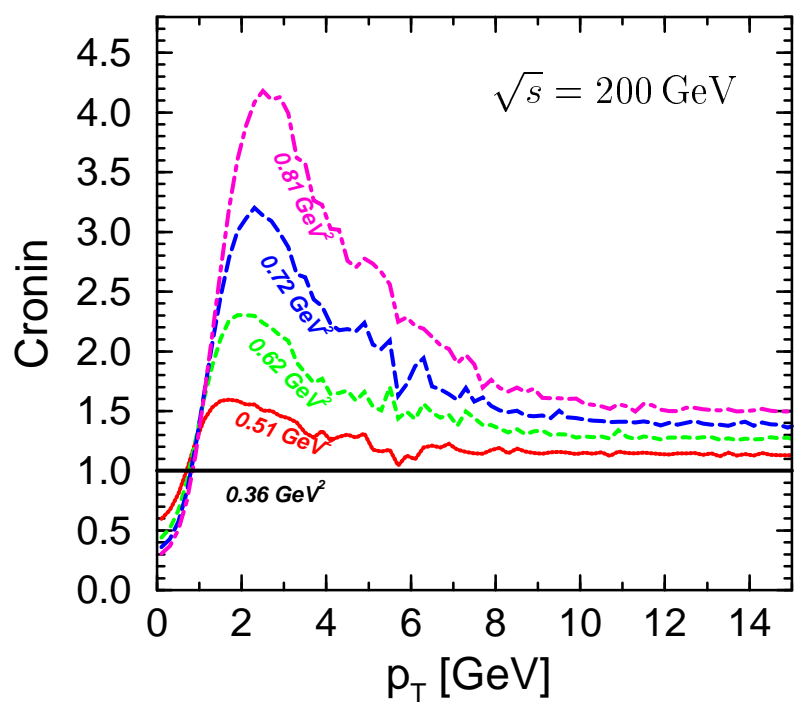

Fig. 4. The Cronin enhancement factor (see text) as a function of the transverse momentum $p_{\perp}$ of charged hadrons for different average $\left\langle k_{\perp}^{2}\right\rangle$ according to the PYTHIA calculations for $\mathrm{N}+\mathrm{N}$ collisions at $\sqrt{s}=200 \mathrm{GeV}$.

which are a function of $\left\langle k_{\perp}^{2}\right\rangle$ and the hadron species. We note, that these 'Cronin enhancement factors' enter the dynamical transport calculations for each hadron type separately, whereas only the average enhancement factor is displayed for charged hadrons in Fig. 4. 
The effect of such Cronin enhancement is obvious from Fig. 4: at low transverse momentum one observes a suppression of the $p_{\perp}$ spectrum with increasing $\left\langle k_{\perp}^{2}\right\rangle$, which leads to a hardening of hadron spectra from nucleus-nucleus collisions relative to $\mathrm{p}+\mathrm{p}$ reactions for transverse masses below about $\left(m_{\perp}-m_{0}\right) \leq$ $1 \mathrm{GeV}$. A maximum in the enhancement factor arises at $p_{\perp} \sim 2 \ldots 3 \mathrm{GeV} / \mathrm{c}$ followed by a smooth decrease towards unity at high transverse momenta, but a sizeable (constant) enhancement (above unity) remains up to the highest momenta considered in our calculations.

\section{$2.4 d+$ Au collisions}

Whereas elementary $\mathrm{p}+\mathrm{p}$ collisions give pseudorapidity distributiuons $d N_{x} / d \eta$ of produced hadrons $x$ symmetric in $\eta$ (Fig. 5), the recent measurements of $d N_{x} / d \eta$ of primary charged hadrons in minimum bias $\mathrm{d}+\mathrm{Au}$ collisions at $\sqrt{s}=200 \mathrm{GeV}$ from the PHOBOS collaboration [50] show a significant asymmetry in $\eta$ (Fig. 5). This asymmetry in $\eta$ is quite well reproduced by our calculations for minimum bias $\mathrm{d}+\mathrm{Au}$ collisions (Fig. 5) as well as the approximate maximum in $d N / d \eta$ for $\eta \approx-2$. This implies that the rescattering of

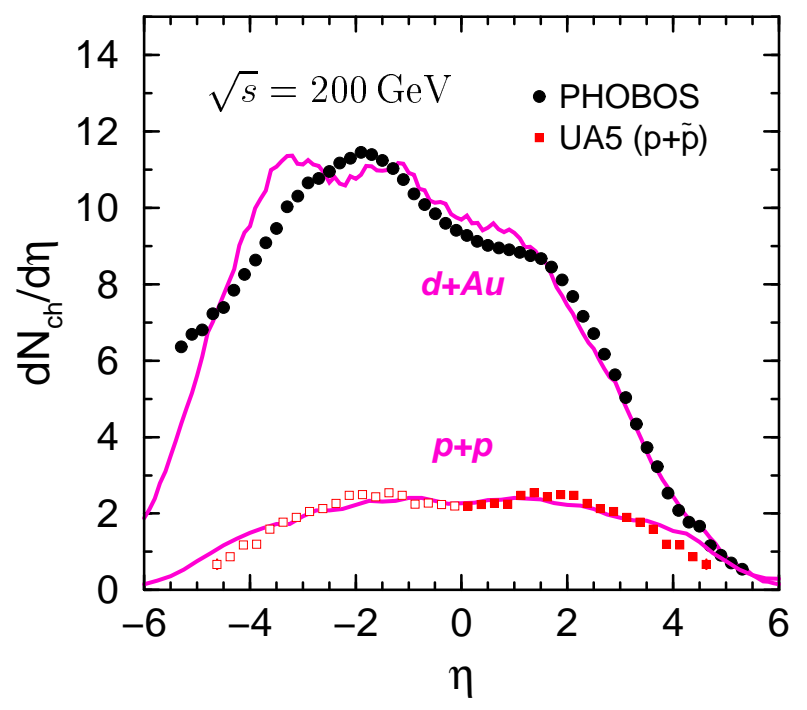

Fig. 5. The calculated pseudorapidity distributions $d N / d \eta$ of charged particles from inelastic $\mathrm{p}+\mathrm{p}$ and $\mathrm{d}+\mathrm{Au}$ reactions at $\sqrt{s}=200 \mathrm{GeV}$ (solid lines) in comparison with the data for $\mathrm{p}+\overline{\mathrm{p}}$ from the UA5 Collaboration [49] and $\mathrm{d}+\mathrm{Au}$ collisions from the PHOBOS Collaboration [50]. [The experimental systematic errors for $\mathrm{d}+\mathrm{Au}$ are on the level of $20 \%$, whereas those in the normalization of our transport calculations are less than $10 \%$.]

pre-hadrons from the deuteron projectile $(\eta>0)$ with nucleons of the target $(\eta<0)$ is essential and that particles with large longitudinal momentum $(\eta \geq 2)$ are absorbed substantially in the Au-target ending up at pseudorapidity ranges closer to the target pseudorapidity. 
In order to sustain these findings, Fig. 5 shows also the comparison of our model calculations for $\mathrm{p}+\mathrm{p}$ with data for $\mathrm{p}+\overline{\mathrm{p}}$ from UA5 [49] at the same invariant energy. The difference between the data and the calculations is on the $10 \%$ level and partly due to the fact, that UA5 measures inelastic events for $\mathrm{p}+\overline{\mathrm{p}}$, whereas the calculations are for $\mathrm{p}+\mathrm{p}$ collisions: We attribute the differences for pseudorapidity regions close to those of the initial baryons (antibaryon) to an excess of light quarks in the $\mathrm{p}+\mathrm{p}$ reaction.

Thus, in addition to the comparison in Fig. 1 for the transverse momentum distribution also the (soft) longitudinal hadron production is reasonably well described in our transport approach for elementary $\mathrm{p}+\mathrm{p}$ as well as $\mathrm{d}+\mathrm{Au}$ reactions.

Since the Cronin enhancement factor essentially depends on $\left\langle k_{\perp}^{2}\right\rangle$, which in turn is controlled by the parameter $\alpha$ in eq. (3), we will determine its range by the data on $\mathrm{d}+\mathrm{Au}$ reactions.

A comparison of the calculated ratio

$$
R_{\mathrm{dA}}\left(p_{\perp}\right)=\frac{1 / N_{\mathrm{dA}}^{\text {event }} d^{2} N_{\mathrm{dA}} / d y d p_{\perp}}{\left\langle N_{\text {coll }}\right\rangle / \sigma_{p p}^{\text {inelas }} d^{2} \sigma_{p p} / d y d p_{\perp}}
$$

is shown in Fig. 6 with the respective data for charged hadrons from the PHENIX and STAR collaborations [13,14] using $\alpha=0.25 \cdots 0.4$ (hatched band). In eq. (4) $\left\langle N_{\text {coll }}\right\rangle \approx 8.5$ denotes the number of inelastic nucleon-nucleon collisions per event (for minimum bias collisions), whereas $\sigma_{p p}^{\text {inelas }}$ is the inelastic $\mathrm{p}+\mathrm{p}$ cross section ${ }^{1}$. We find that our calculations give a rise in the ratio eq. (4) for small $p_{\perp}$ and a slight decrease for $p_{\perp} \geq 2.5 \mathrm{GeV} / \mathrm{c}$ which can be traced back to inelastic interactions of the leading pre-hadrons with the nucleons in the target. The description of the data for $R_{\mathrm{dA}}\left(p_{\perp}\right)$ is not perfect and the data even indicate a slightly larger enhancement for $p_{\perp}=4 \ldots 6 \mathrm{GeV} / \mathrm{c}$, but the agreement is sufficient to proceed with $\mathrm{Au}+\mathrm{Au}$ collisions at the same $\sqrt{s}$. The important issue to remember in this context is that no dramatic absorption of high $p_{\perp}$ hadrons in $d+A u$ collisions is found in the calculations as well as in the data.

We note that the number of inelastic collisions $\left\langle N_{\text {coll }}\right\rangle$ entering the ratio eq. (4) are directly available in the transport calculations; thus no model dependence enters the computation of this ratio.

For completeness we show in Fig. 5 also the result for the ratio (4) from a calculation without any initial state Cronin enhancement (solid line). In this

1 We remark with some caution, that the underlying binary scaling according to the Glauber model has recently been questioned by Kopeliovich [51] since inelastic shadowing corrections should reduce the total inelastic cross section by up to $20 \%$. 


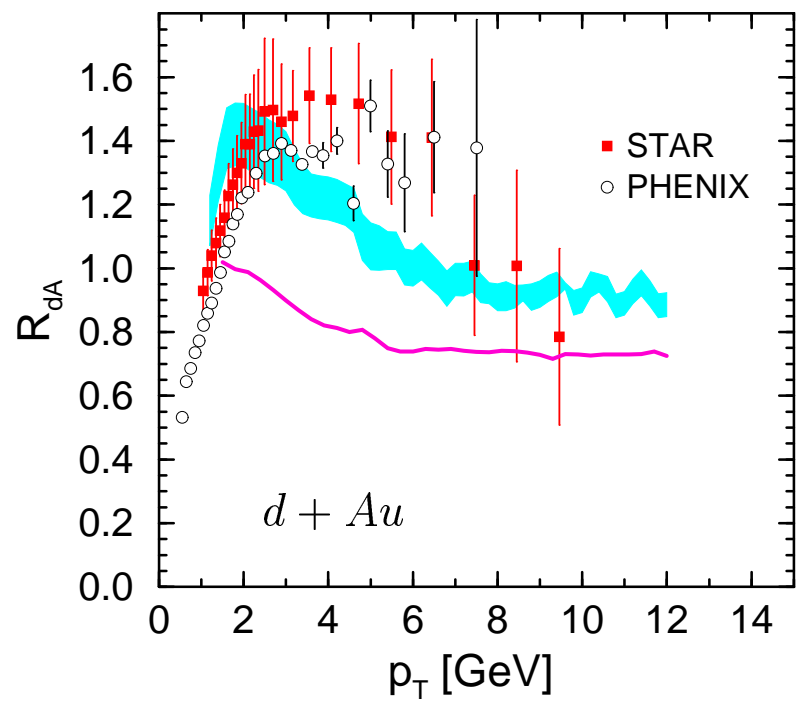

Fig. 6. The suppression factor $R_{\mathrm{dA}}$ for minimum bias $\mathrm{d}+\mathrm{Au}$ collisions $(\sqrt{s}=200 \mathrm{GeV})$ at midrapidity for charged hadrons. Experimental data are from PHENIX [13] (open circles) and STAR [14] (filled squares). The hatched band denotes our calculations for the 'Cronin' parameter $\alpha=0.25 \cdots 0.4$ in (3) while the solid line results from transport calculations without employing any initial state Cronin enhancement $(\alpha=0)$.

limit the ratio drops below unity due to the interactions of the leading prehadrons with the residual target nucleons.

\section{$2.5 A u+A u$ collisions}

As in case of the $\mathrm{d}+\mathrm{Au}$ system we expect also an attenuation of high momentum hadrons in $\mathrm{Au}+\mathrm{Au}$ reactions due to inelastic interactions of leading pre-hadrons with the nucleons from the target (projectile). In addition, inelastic interactions between the pre-hadrons among each other should occur as well as between leading pre-hadrons and formed secondary hadrons. Also interactions of formed secondary hadrons between each other might contribute to the final attenuation of high $p_{T}$ particles, if they are formed sufficiently early inside the hot/dense fireball [21]. We mention, that in the calculations to be shown below there are no interactions between leading pre-hadrons and explicit quark or antiquarks or gluons in the very early phase of the collision.

Furthermore, in the HSD approach the formation of secondary hadrons is not only controlled by the formation time $\tau_{f}$, but also by the energy density in the local rest frame, i.e., hadrons are not allowed to be formed if the energy density is above $1 \mathrm{GeV} / \mathrm{fm}^{3}$ [28]. This energy density cut in HSD prevents hadrons to be formed in central nucleus-nucleus collisions at RHIC for a couple of $\mathrm{fm} / \mathrm{c}$ when setting the clock by the initial impact of the two ions. We note 
in passing, that a reduction of this energy density cut to $0.7 \mathrm{GeV} / \mathrm{fm}^{3}$ (cf. Introduction) leads to a further delay of secondary hadron formation by $\sim 1.2$ $\mathrm{fm} / \mathrm{c}$. Recall, however, that this energy cut does not apply for the pre-hadronic states defined above.

We start with most central (5\% centrality) $\mathrm{Au}+\mathrm{Au}$ collisions at $\sqrt{s}=200 \mathrm{GeV}$. In this case the nuclear modification factor is defined in accordance with eq. (4) as

$$
R_{\mathrm{AA}}\left(p_{\perp}\right)=\frac{1 / N_{\mathrm{AA}}^{\text {event }} d^{2} N_{\mathrm{AA}} / d y d p_{\perp}}{\left\langle N_{\text {coll }}\right\rangle / \sigma_{p p}^{\text {inelas }} d^{2} \sigma_{p p} / d y d p_{\perp}} .
$$

Fig. 7 shows a comparison of the calculations for eq. (5) with the data for charged hadrons from Ref. [10,11], where the hatched band again corresponds to the range in the parameter $\alpha$ in eq. (3) from $0.25 \ldots 0.4$. The calculations

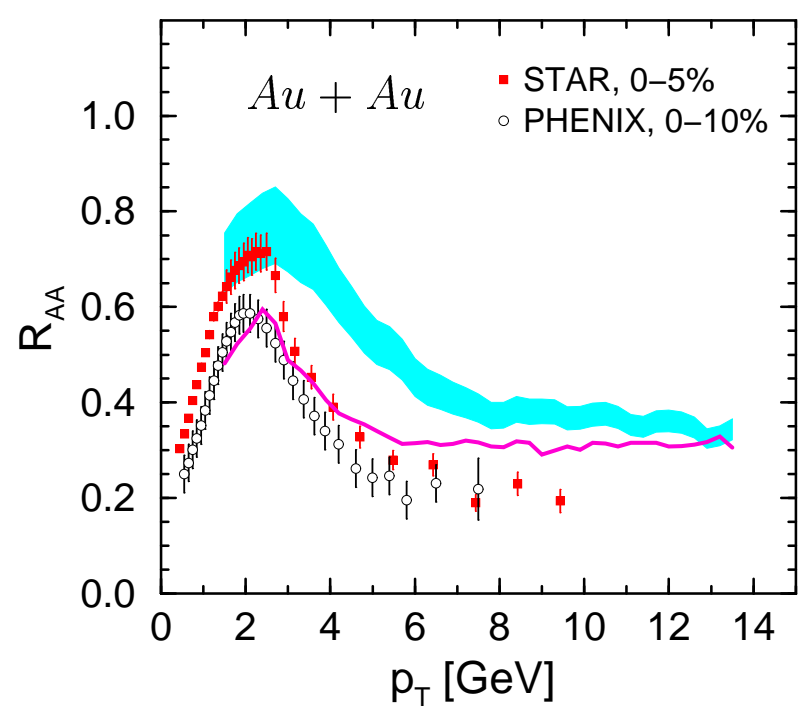

Fig. 7. The suppression factor $R_{\mathrm{AA}}$ of charged hadrons at $0 \cdots 5 \%$ central $\mathrm{Au}+\mathrm{Au}$ collisions $(\sqrt{s}=200 \mathrm{GeV})$ at midrapidity; Experimental data are from Refs. [10,11]. Please note, that the PHENIX data are for $0 \cdots 10 \%$ centrality. The hatched band denotes our calculations for the 'Cronin' parameter $\alpha=0.25 \cdots 0.4$ in (3). The solid line results from transport calculations without employing any initial state Cronin enhancement $(\alpha=0)$.

roughly reproduce the shape of the ratio $R_{\mathrm{AA}}\left(p_{\perp}\right)$ but overestimate the experimental ratio at higher $p_{\perp}$. We emphasize, that the Cronin enhancement is visible at all momenta, but does not show up to be responsible for the peak structure in the enhancement around $2 \mathrm{GeV} / \mathrm{c}$. This becomes clear by comparing to the lower solid line, which results from transport calculations without employing any initial state Cronin enhancement. In this case the ratio $R_{\mathrm{AA}}\left(p_{\perp}\right)$ is slightly low at $p_{\perp} \approx 2 \ldots 3 \mathrm{GeV} / \mathrm{c}$ in comparison to the data from the STAR collaboration, but rather well in line with the data from PHENIX. 
For $p_{\perp}>5 \mathrm{GeV} / \mathrm{c}$, however, it still underestimates the suppression seen by both collaborations.

In order to understand this result we decompose the ratio $R_{\mathrm{AA}}$ into contributions from pions, kaons and $\rho, \omega, K^{*}$ vector mesons (before decays) without employing any initial state Cronin enhancement. The resulting comparison is shown in the left part of Fig. 8 for $5 \%$ central $\mathrm{Au}+\mathrm{Au}$ collisions. Here the
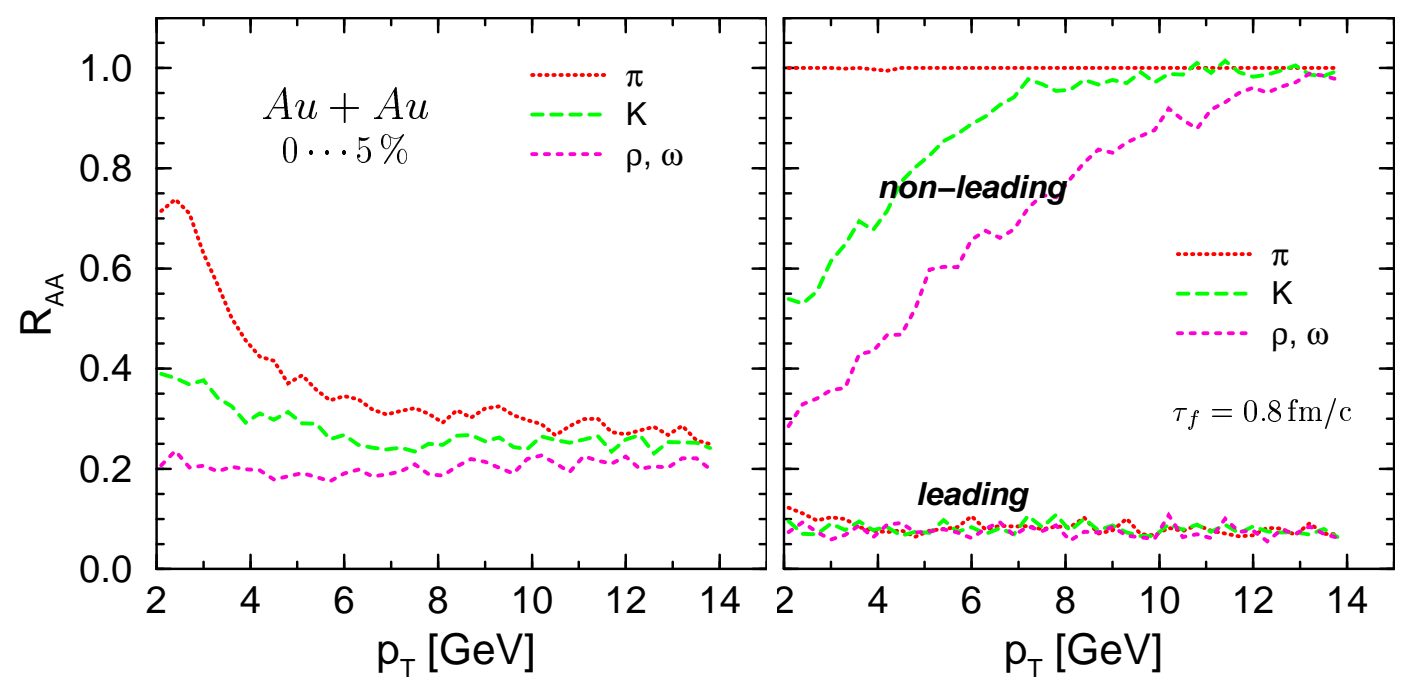

Fig. 8. The nuclear modification factor for central $\mathrm{Au}+\mathrm{Au}$ collisions $(\sqrt{s}=200 \mathrm{GeV})$ prior to any particle decays. The l.h.s. shows the total theoretical ratios for the particle classes $\pi, \rho, \omega$ and $K, K^{*}$. The right panel separates the theoretical contributions of the left panel into the 'leading' and the 'non-leading' particle contributions.

direct pions show a reduced attenuation, the kaon reduction is slightly larger for lower $p_{\perp}$, while the vector meson absorption is much stronger. Hadron formation time effects apparently play a substantial role in the few $\mathrm{GeV} / \mathrm{c}$ region since heavier hadrons are formed earlier than light pions in the cms frame at fixed transverse momentum due to the lower Lorentz boost.

This expectation is quantified in the right part of Fig. 8 where the decomposition of the ratio $R_{\mathrm{AA}}$ is additionally performed for leading and secondary/nonleading hadrons. First of all, the attenuation of the leading pions, kaons and vector mesons is roughly independent on $p_{\perp}$ and hadron type and gives $R_{\mathrm{AA}} \approx 0.09$. Thus the dominant attenuation seen experimentally should be addressed to the interactions of leading (unformed) pre-hadrons. This becomes even more apparent in accordance with all "back-of-the-envelope" estimates (including a constant rest frame formation time $\tau_{f}$ ) when looking at the suppression factors for the non-leading hadrons in Fig. 8 (right part). Here the direct pions practically do not show any attenuation in line with the expectation from the large formation times of pions with momenta of a couple of $\mathrm{GeV} / \mathrm{c}$. The situation changes for kaons and especially vector mesons due to their larger 
mass. Now formed hadrons of a few $\mathrm{GeV} / \mathrm{c}$ may interact with other formed hadrons and look 'thermalized', i.e. their final momenta show up in the soft part of the spectrum (below $p_{\perp} \approx 2 \mathrm{GeV} / \mathrm{c}$ ). Since the expanding fireball has the same geometrical shape for all high $p_{\perp}$ hadrons, the attenuation in this "constant $\tau_{f}$ " picture is intimately correlated with the Lorentz $\gamma$-factor of the particles such that for high $p_{\perp}$ mesons above about $6 \ldots 8 \mathrm{GeV} / \mathrm{c}$ no absorption of hadrons should occur via interactions of formed hadrons. On the other hand, the final attenuation of high $p_{\perp}$ hadrons is approximately independent on the actual formation time because according to Fig. 3 these particles are essentially leading pre-hadrons that rescatter anyhow.

In order to demonstrate the effect of the formation time $\tau_{f}$ we show in Fig. 9 our results for the attenuation of secondary pions, kaons and vector mesons for $\tau_{f}=0.5 \mathrm{fm} / \mathrm{c}$. The latter value for $\tau_{f}$ is even favored by studies on antiproton

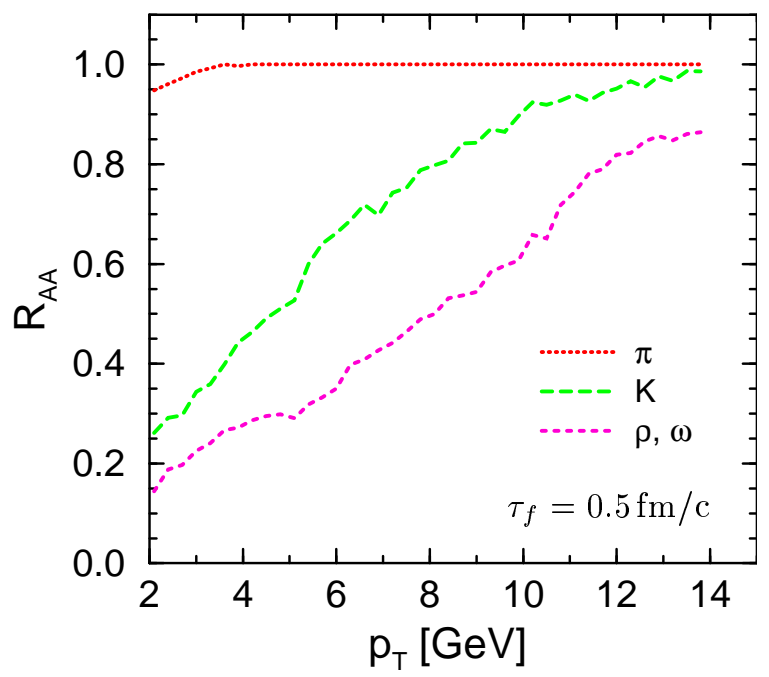

Fig. 9. The same as Fig. 8 (right panel) for a formation time $\tau_{f}=0.5 \mathrm{fm} / \mathrm{c}$. (Only the ratios of non-leading hadrons are displayed.)

production and reabsorption in $\mathrm{p}+\mathrm{A}$ reactions at AGS energies [45] as well as hadron attenuation in virtual photoproduction off nuclei [43]. In line with the discussion above we see from Fig. 9 - in comparison to the right part of Fig. 8 - that the suppression of kaons and vector mesons increases for a lower formation time and also extends to higher $p_{\perp}$. However, as noted above, the total attenuation is essentially determined by the fraction of leading prehadrons at fixed $p_{\perp}$ (Fig. 3) and by their effective cross section with baryons or mesons. Formation time effects can only show up at lower $p_{\perp}$.

We note in passing that a decrease in the energy density cut from $1 \mathrm{GeV} / \mathrm{fm}^{3}$ to $0.7 \mathrm{GeV} / \mathrm{fm}^{3}$ in the HSD calculations with respect to the formation of secondary hadrons leads only to a minor modification (enhancement) of the ratio $R_{A A}\left(p_{\perp}\right)$ by about $10 \%$, i.e. for $p_{\perp} \geq 6 \mathrm{GeV} /$ c the ratio increases from $\sim 0.4$ to $\sim 0.45$. 
We now turn to an alternative model for the leading pre-hadron cross section, which is discussed in the literature [52] (cf. also Ref. [21]) since the notion of a fractional constant cross section might be questionable [35] and alternative assumptions should be tested. ${ }^{2}$ To this aim we have adopted a time-dependent cross section for leading pre-hadrons of the kind

$$
\sigma_{\text {lead }}(\sqrt{s})=\frac{t-t_{0}}{t_{f}} \sigma_{\text {had }}(\sqrt{s})
$$

for $t-t_{0} \leq t_{f}$, where $t_{0}$ denotes the actual production time and $t_{f}$ its formation time in the calculational frame. The full hadronic cross section is adopted for $t \geq t_{0}$. The numerical results of this assumption are shown for the ratio $R_{\mathrm{AA}}$ in Fig. 10 in case of $5 \%$ central $\mathrm{Au}+\mathrm{Au}$ collisions. We find that the concept eq. (6)

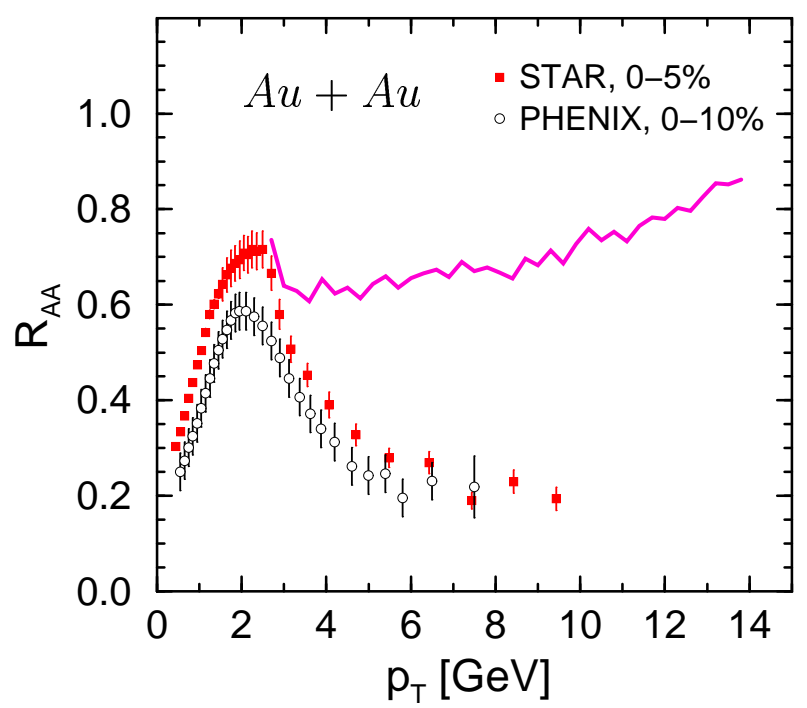

Fig. 10. Same as fig. 7, but with a leading cross section according to eq. (6) for the perturbative high $p_{\perp}$ particles.

is not in accordance with the experimental observation since the leading prehadron cross section is initially too low, i.e. when the fireball is very compact and dense. Furthermore, assuming eq. (6) in the nonperturbative transport calculations leads to dramatic consequences: In this case the pion, kaon and antibaryon rapidity distributions are severely underestimated due to the lack of inelastic reactions from leading pre-hadrons during the passage time of the heavy nuclei.

We thus continue our analysis with the 'default' formation time $\tau_{f}=0.8 \mathrm{fm} / \mathrm{c}$ and leading pre-hadron cross sections in line with the constituent quark model

2 In the string fragmentation picture (combined with the constituent quark model) a considerable separation in space for the string ends at the starting time is unavoidable. The only possible pointlike structure here is the hard scattered quark from a string end. 
without attempting to 'fine-tune' any parameter.

\subsection{Dependence on centrality}

The centrality dependence of the ratio $R_{\mathrm{AA}}$ is shown in Fig. 11 for $20 \cdots 30 \%$, $30 \cdots 40 \%, 40 \cdots 60 \%$ and $60 \cdots 80 \%$ centrality of $\mathrm{Au}+\mathrm{Au}$ collisions at $\sqrt{s}=$ $200 \mathrm{GeV}$. Again the hatched bands correspond to our calculations with a
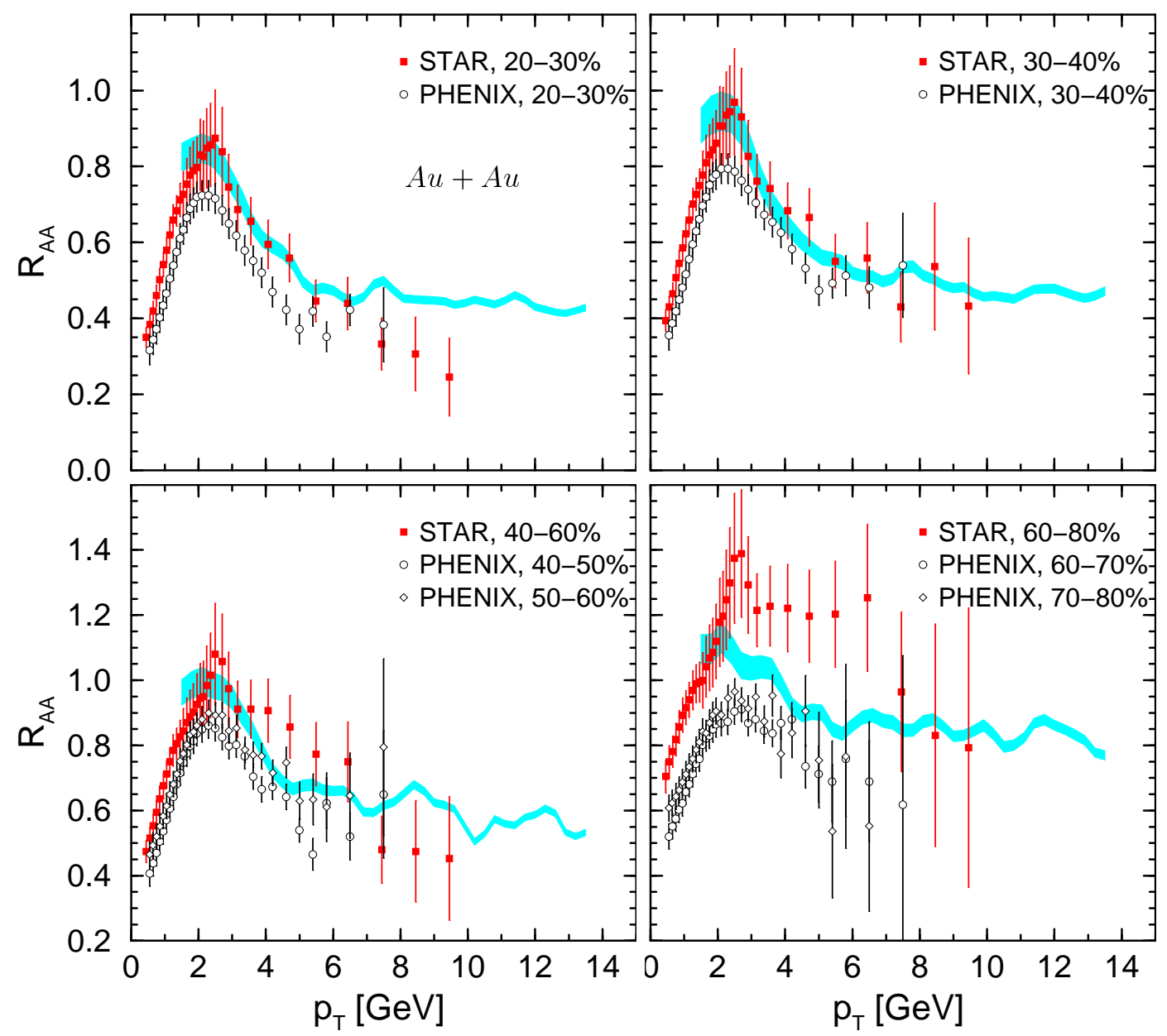

Fig. 11. Same as Fig. 7 for the centralities $20 \cdots 30 \%, 30 \cdots 40 \%, 40 \cdots 60 \%$ and $60 \cdots 80 \%$. Calculational fluctuations are due to limited statistics. Experimental data are from Refs. [11,10].

Cronin parameter $\alpha$ in (3) ranging from 0.25 to 0.4 while the data stem from Refs. $[11,10]$. Note, that the uncertainty in the Cronin enhancement (width of the hatched band) decreases for more peripheral reactions, which is a direct consequence of the lower number of hard $N N$ collisions in (3). Our calculations turn out to be in a better agreement with the data - or interpolate between the measurements from the STAR and PHENIX collaborations for 60 to $80 \%$ centrality - than for the most central $\mathrm{Au}+\mathrm{Au}$ collisions discussed in the 
previous subsection. An analysis with respect to various contributions as in Fig. 7 shows, that with decreasing centrality the influence of formed hadrons essentially vanishes in the ratio $R_{\mathrm{AA}}\left(p_{\perp}\right)$ and the final state suppression is dominated by the interaction of leading pre-hadrons either 'immediately' with the baryons or other leading debries of the colliding nuclei or with the hadrons formed at later times.

In order to obtain a measure for the centrality dependence of the suppression we display in Fig. 12 the suppression factor $\left\langle R_{\mathrm{AA}}\right\rangle$ for charged hadrons integrated for $p_{\perp} \geq 4.5 \mathrm{GeV} / \mathrm{c}$ in comparison to the same quantity from the PHENIX collaboration [10] as a function of the number of participating nucleons $N_{\text {part }}$. We mention that the determination of $N_{\text {part }}$ is model dependent

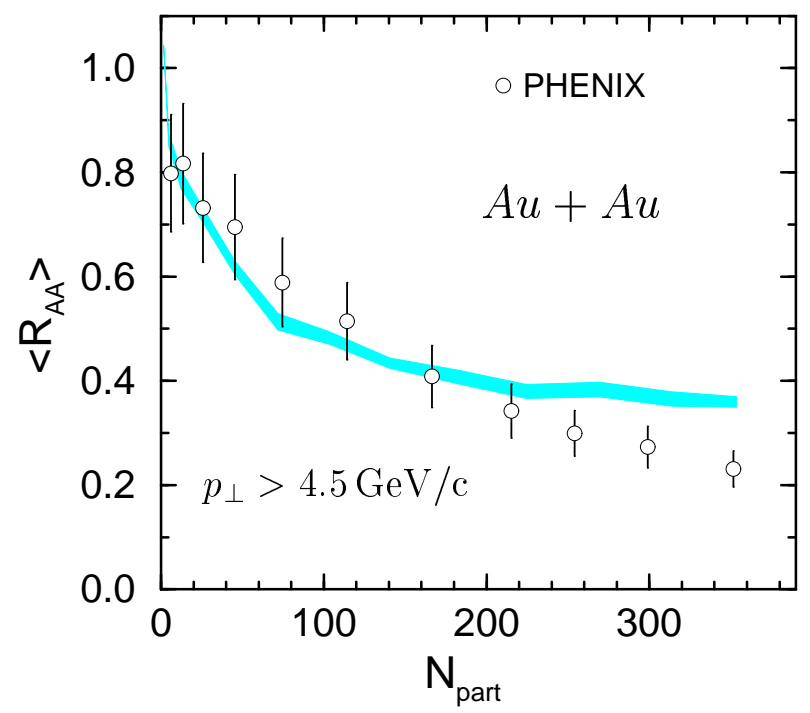

Fig. 12. The ratio $\left\langle R_{A A}\right\rangle$ for charged hadrons for $p_{\perp} \geq 4.5 \mathrm{GeV} / \mathrm{c}$ as a function of $N_{\text {part }}$ in comparison to the data from Ref. [10].

for very peripheral reactions, but rather save for mid-central and central reactions. As already seen from Figs. 10 we describe the average suppression factors well for $N_{\text {part }} \leq 150$ and underestimate the suppression for central $\mathrm{Au}+\mathrm{Au}$ interactions.

It is worth pointing out that the effective cross section of the leading prehadrons is constant during their propagation and thus the suppression is linear in the effective propagation length $L$. As shown in a geometrical model by Drees et al. [53] this linear absorption mechanism - when fixed in strength to the most central collisions - overestimates the amount of suppression for mid-central reactions. According to Ref. [53] the absorption geometry favors a suppression $\sim L^{2}$ in comparison to the data in Fig. 12. Though we do not find a strong argument in favor of any $\sim L^{2}$ suppression mechanism for all centrality classes we, nevertheless, seem to need some additional attenuation for central $\mathrm{Au}+\mathrm{Au}$ reactions which could be attributed to strong interactions of the leading pre-hadrons in a 'colored' medium. 


\subsection{Elliptic flow and Angular Correlations}

Since the leading pre-hadron cross section employed in our transport calculation is much larger than related cross sections from pQCD, shadowing phenomena, that are related to the initial geometry in coordinate space for fixed impact parameter $b$, should show up in angular correlations like the elliptic flow of the high $p_{\perp}$ hadrons. In fact, a rather high and approximately constant elliptic flow has been seen experimentally for $p_{\perp} \geq 2 \mathrm{GeV} / \mathrm{c}$. It is still to be answered, whether this elliptic flow is a consequence of suppression due to strongly interacting matter or a survivor of correlations from the hard nucleon-nucleon interactions and following fragmentations.

Gating on particles with momenta $p_{\perp} \geq 4 \mathrm{GeV} / \mathrm{c}$ in the transport calculation, only $1 / 3$ of these final hadrons have suffered one or more interactions during their propagation to the vacuum, whereas the other $2 / 3$ escape without any interaction. This observation implies, since more than $3 / 4$ of the high $p_{\perp}$ hadrons are strongly absorbed, that the final high $p_{T}$ hadrons seen experimentally essentially stem from pre-hadrons that originate from a diffuse 'surface region' of the expanding fireball. The latter pre-hadrons then evolve (or fragment) to the final hadrons dominantly in the vacuum and are accompanied by secondary hadrons, which - due to their large formation time - also hadronize in the vacuum. Thus, when gating on high $p_{\perp}$ hadrons (in the vacuum) the 'near-side' correlations should be close to the 'near-side' correlations observed for jet fragmentation in the vacuum. According to our understanding the absence of 'near-side' jet broadening does not necessarily indicate the presence of radiative quark energy loss in a QGP phase as advocated in Ref. [54].

We emphasize, that within our calculations the particles with large transverse momenta - that are treated perturbatively - so far have no intrinsic correlations as characteristic for back-to-back jet correlations. This point will be addressed in the near future within a separate study.

The elliptic flow defined by

$$
v_{2}\left(p_{\perp}\right)=\left\langle\frac{p_{x}^{2}-p_{y}^{2}}{p_{x}^{2}+p_{y}^{2}}\right\rangle_{p_{\perp}}
$$

provides a measure for the coupling of degrees of freedom in coordinate space with those in momentum space. In eq. (7) the $y$-direction denotes in-plane motion whereas the $x$-direction denotes out-of-plane propagation. At RHIC energies a strong in-plane elliptic flow is seen experimentally which for $p_{\perp} \leq$ $1.5 \mathrm{GeV} / \mathrm{c}$ is rather well described by hydrodynamical calculations [55]. However, hydrodynamical calculations overestimate the approximately constant elliptic flow for higher transverse momenta. This observation proves that the 
medium created in $\mathrm{Au}+\mathrm{Au}$ collisions is strongly interacting and the cross section needed to generate such a high elliptic flow is much larger than expected from standard kinetic pQCD [56].

In Fig. 13 we show the calculated elliptic flow $\left\langle v_{2}\right\rangle$ for charged hadrons with transverse momentum $p_{\perp}=2 \cdots 6 \mathrm{GeV} / \mathrm{c}$ in comparison to the data from Ref. [57]. One observes that our calculations underestimate the data by more

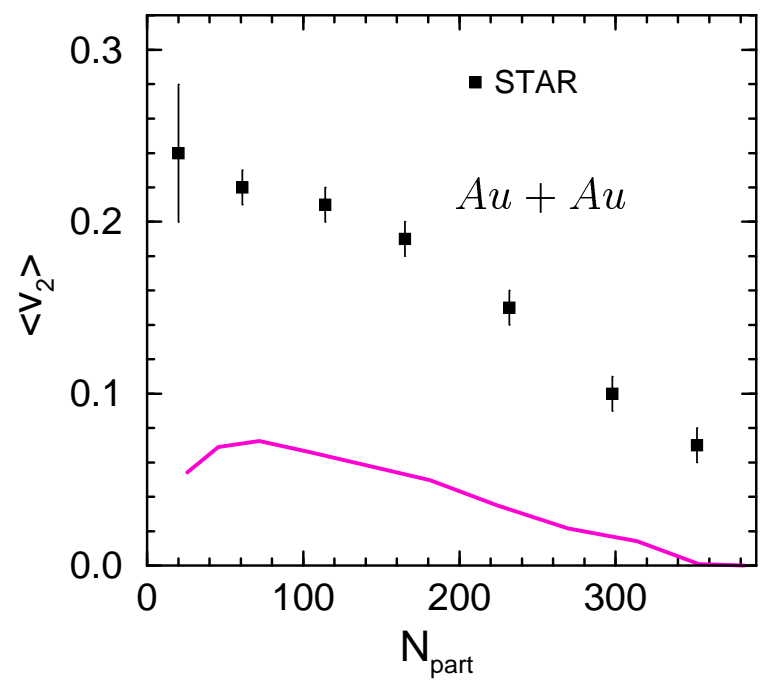

Fig. 13. The elliptic flow $\left\langle v_{2}\right\rangle$ for charged hadrons for $p_{\perp}=2 \cdots 6 \mathrm{GeV} / \mathrm{c}$ as a function of $N_{\text {part }}$ in comparison to the data from Ref. [57].

than a factor of 3 ; however, it is presently not clear if non-flow contributions of different origin contribute to the measured $v_{2}$ values. The elliptic flow described in our approach is essentially due to a shadowing of high $p_{\perp}$ hadrons in the out-of-plane direction, since there are no contributions from a collective pressure in the perturbative dynamics of the high $p_{\perp}$ hadrons. As already addressed by Shuryak [58], such shadowing effects should be too weak to describe the large $v_{2}$ values seen experimentally. The authors of Ref. [53] come to a very similar conclusion in their geometrical model (cf. also Ref. [54]).

\subsection{SPS energies}

In this subsection we briefly comment on results obtained in central collisions of $\mathrm{Pb}+\mathrm{Pb}$ at $\sqrt{s}=17.3 \mathrm{GeV}$, i.e. at top SPS energies. We mention - without explicit representation - that the midrapidity $p_{\perp}$ spectra of hadrons from our PYTHIA calculations are approximately exponential in the transverse momentum showing no explicit power-law shape as for $\sqrt{s}=200 \mathrm{GeV}$ in Fig. 1. This result stems in part from the kinematical cut in the maximum transverse momentum, which is about $7 \mathrm{GeV} / \mathrm{c}$ at $\sqrt{s}=17.3 \mathrm{GeV}$ since two strings (jets) have to be formed. Furthermore, a direct comparison to related experimental 
spectra is not possible because such data have not been measured. One might extrapolate the hadron $p_{\perp}$ spectra from higher energies assuming a $x_{\perp}$ scaling law to get a phenomenological parametrisation as in Ref. [59], however, our independent attempt in this direction showed systematic, non-constant errors (in important parts of the spectra) up to a factor of 2 when employing this ansatz. This uncertainty has to be kept in mind especially when comparing $p_{\perp}$ spectra from central $\mathrm{Pb}+\mathrm{Pb}$ collisions to scaled 'extrapolated' spectra from $\mathrm{p}+\mathrm{p}$ reactions at the same $\sqrt{s}$.

Since at $\sqrt{s}=17.3 \mathrm{GeV}$ the kinematical limits become essential for high $p_{\perp}$ hadrons, the ratio of 'leading particles' to 'all produced particles' (before particle decays) in $\mathrm{N}+\mathrm{N}$ collisions changes considerably in comparison to Fig. 3 for $\sqrt{s}=200 \mathrm{GeV}$. This information is displayed in Fig. 14 for different particle classes as a function of transverse momentum as calculated within our PYTHiA description. A direct consequence of the results in Fig. 14 is that

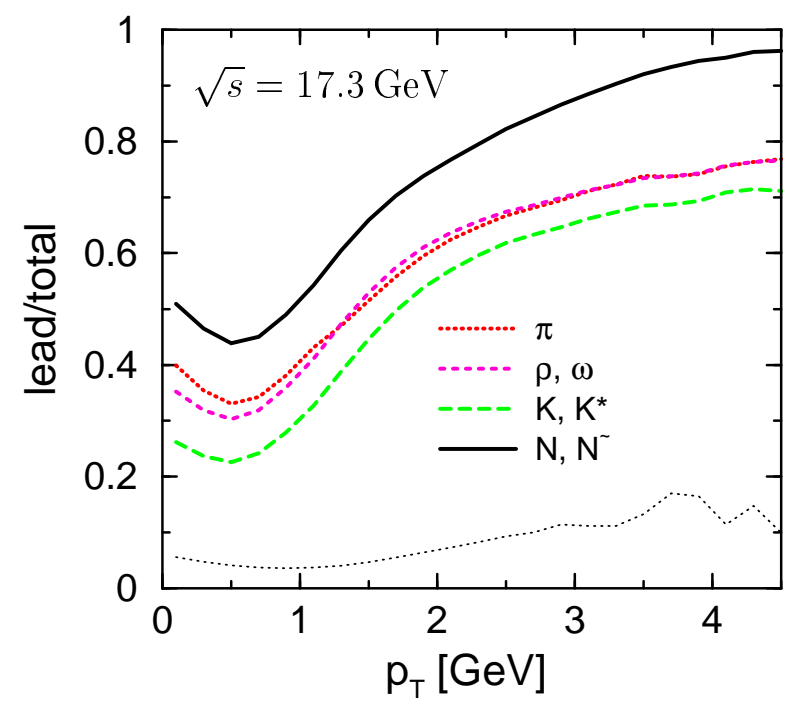

Fig. 14. Same as Fig. 3 for $\sqrt{s}=17.3 \mathrm{GeV}$. (Note the different momentum scales.) The thin dotted line around 0.1 shows the contribution of leading baryons with 2 leading quarks.

above $\sim 2 \ldots 3 \mathrm{GeV} / \mathrm{c}$ now most of the mesons and the major fraction of nucleons are of leading origin and - within the dynamics specified above - may interact without delay.

A further consequence of the steeper transverse momentum spectra at $\sqrt{s}=$ $17.3 \mathrm{GeV}$ relative to $\sqrt{s}=200 \mathrm{GeV}$ (Fig. 1) is that we get a larger Cronin enhancement for the SPS energy. This effect is due to a more drastic change in the spectra when enhancing $\left\langle k_{\perp}^{2}\right\rangle$ in the string fragmentation; as a consequence the ratio between a calculation with enhanced $\left\langle k_{\perp}^{2}\right\rangle$ to a calculation with the default value for $\mathrm{p}+\mathrm{p}$ collisions becomes larger as seen from Fig. 15. This implies that the initial state Cronin enhancement is larger than at RHIC energies (cf. Fig. 4). 


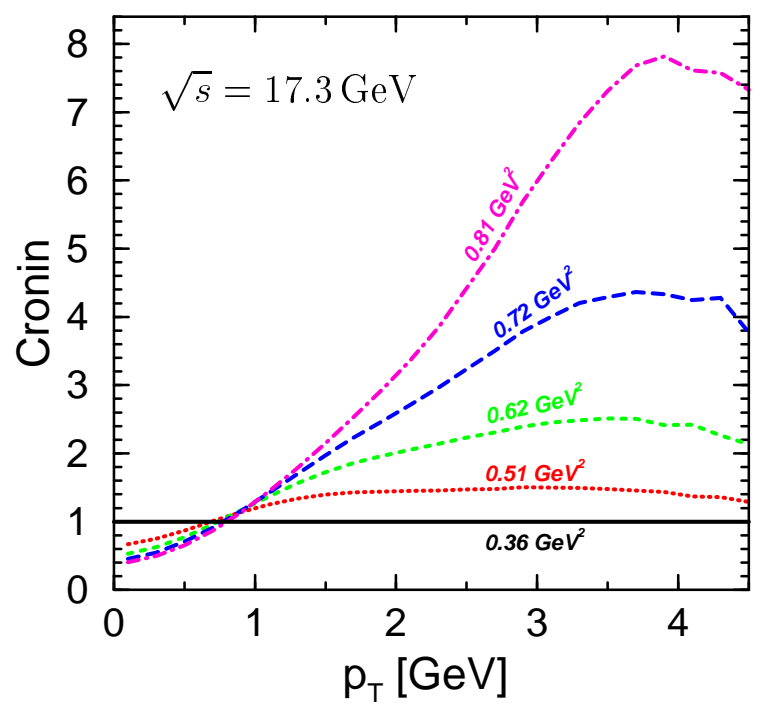

Fig. 15. Same as Fig. 4 for $\sqrt{s}=17.3 \mathrm{GeV}$. (Note the different momentum scales and also the different sizes.)

Fig. 16 shows the result of our transport calculations for the ratio (5) in $5 \%$ central $\mathrm{Pb}+\mathrm{Pb}$ collisions at $\sqrt{s}=17.3 \mathrm{GeV}$, where the hatched band again corresponds to the range in the parameter $\alpha$ in eq. (3) from $0.25 \cdots 0.4$. As

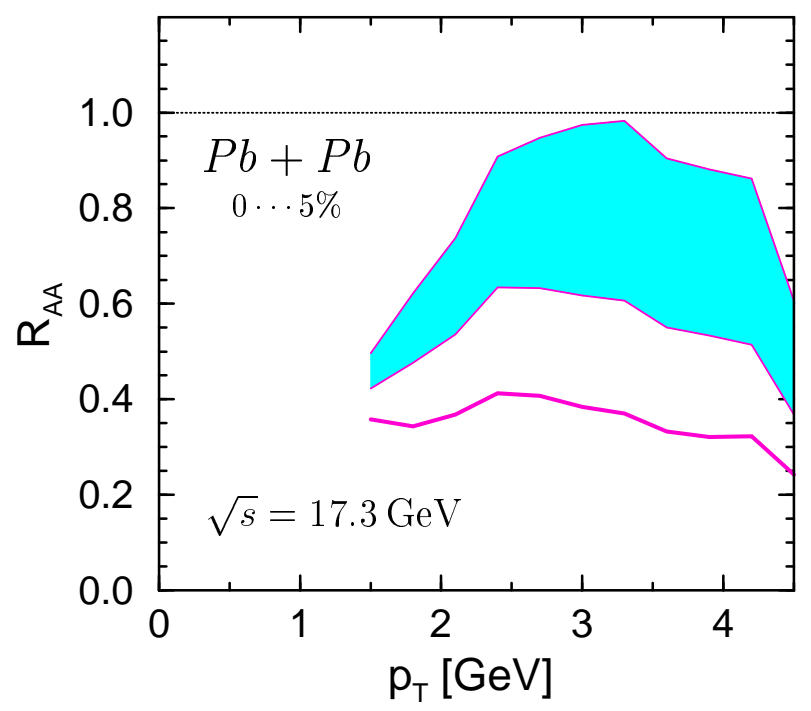

Fig. 16. The suppression factor $R_{\mathrm{AA}}$ of charged hadrons at $0 \cdots 5 \%$ central $\mathrm{Pb}+\mathrm{Pb}$ collisions $(\sqrt{s}=17.3 \mathrm{GeV})$ at midrapidity. The hatched band corresponds to our calculations with the parameter $\alpha$ in (3) ranging from 0.25 to 0.4 . The solid line results from transport calculations without employing any initial state Cronin enhancement $(\alpha=0)$.

in Fig. 6 the solid line shows a calculation without any initial state Cronin enhancement. We see from Fig. 16, that the uncertainty in the ratio (5) due to missing constraints on the initial state effects becomes large. The underlying physics, however, is the same as at RHIC energies: the leading high $p_{\perp}$ 
pre-hadrons interact with the baryons of the projectile/target as well as with secondary formed hadrons. This leads to a net ratio of $\sim 0.35$ for $p_{\perp} \geq 2 \mathrm{GeV} / \mathrm{c}$ when discarding any initial state enhancement (solid line in Fig. 16). However, this large attenuation is counterbalanced by a strong initial state Cronin enhancement, which leads to an increase of the ratio (5) up to $2.5 \ldots 3 \mathrm{GeV} / \mathrm{c}$ followed by a moderate decrease.

We have to mention that the WA98 collaboration has reported on the ratio $R_{\mathrm{AA}}$ for neutral pions at top SPS energies [59] using extrapolations for the $\pi^{0}$ spectra from $\mathrm{p}+\mathrm{p}$ reactions taken from $\pi^{0}$ (and $\pi^{ \pm}$) spectra at larger energies. They quote a steep increase of $R_{\mathrm{AA}}$ with $p_{\perp}$ up to transverse momenta of $3.5 \mathrm{GeV} / \mathrm{c}$ (although with large error bars), which is not described by our transport calculations. However, in view of the uncertainties in the normalization of the ratio (and the ratio itself!) we have to wait for measurements at RHIC for $\sqrt{s} \approx 20 \mathrm{GeV}$, where the transverse momentum spectra from $\mathrm{p}+\mathrm{p}$, $\mathrm{d}+\mathrm{Au}$ and central $\mathrm{Au}+\mathrm{Au}$ collisions can be measured by the same detector setup at the same $\sqrt{s}$ in order to clarify this issue from the experimental side.

\section{Summary}

Summarizing this study, we point out that (pre-) hadronic final state interactions, as included by default in present transport approaches like HSD, are able to approximately reproduce the high $p_{\perp}$ suppression effects observed in $\mathrm{d}+\mathrm{Au}$ and $\mathrm{Au}+\mathrm{Au}$ collisions at RHIC $(\sqrt{s}=200 \mathrm{GeV})$. This finding is remarkable, since the same dynamics also describe the hadron formation and attenuation in deep-inelastic lepton scattering off nuclei at HERMES [43] quite well. Additionally, this also holds for antiproton production and attenuation in proton-nucleus collisions at AGS energies [45].

We have, furthermore, found that interactions of formed hadrons after a formation time $t_{f}$ (which should be a matter of further debate) are not able to explain the attenuation observed experimentally for transverse momenta $p_{\perp} \geq 6 \mathrm{GeV} /$ c. However, the shape of the ratio $R_{\mathrm{AA}}$ in transverse momentum $p_{\perp}$ reflects the presence of final state interactions of formed hadrons in the $1 \ldots 5 \mathrm{GeV} / \mathrm{c}$ range $[21]$.

In order to describe the experimental data below $p_{\perp} \simeq 5 \mathrm{GeV} / \mathrm{c}$, a reasonable description of the initial state "Cronin effect" has to be taken into account, which has been modeled in this work by increasing the average $\left\langle k_{\perp}^{2}\right\rangle$ of the string fragmentation in subsequent hard collisions and has been fixed approximately by the $\mathrm{d}+\mathrm{Au}$ measurements at RHIC. Such initial state effects are of pre-hadronic origin in a strongly interacting medium. Since they also show up in energetic proton-nucleus reactions $[46,47]$, the initial state effects cannot 
be considered as an indication for a phase transition (or crossover) of the high density matter produced in $\mathrm{Au}+\mathrm{Au}$ collisions at relativistic energies.

We have demonstrated, that the centrality dependence of the modification factor $R_{\mathrm{AA}}$ in $\mathrm{Au}+\mathrm{Au}$ collisions at $\sqrt{s}=200 \mathrm{GeV}$ is well described for peripheral and mid--central collisions on the basis of leading pre-hadron interactions. However, the attenuation in central $\mathrm{Au}+\mathrm{Au}$ collisions is underestimated, which one may address to additional interactions of partons in a colored medium that have not been accounted for in our present transport studies.

We finally note, that the elliptic flow $v_{2}$ for high transverse momentum particles is underestimated by at least a factor of 3 (cf. Fig. 13). This demonstrates that geometrical shadowing in out-of-plane direction is not sufficient to get the right magnitude of the elliptic flow seen experimentally, which supports the independent studies in Refs. [58,53].

Since the high $p_{\perp}$ particles in the present work have no intrinsic back-toback jet-like correlations, we postpone any further detailed statements to an upcoming investigation in the near future. Moreover, further experimental studies on the suppression of high momentum hadrons from $\mathrm{d}+\mathrm{Au}$ and $\mathrm{Au}+\mathrm{Au}$ collisions down to $\sqrt{s}=20 \mathrm{GeV}$ will be necessary to separate initial state Cronin effects from final state attenuation and to disentangle to role of partons in a colored partonic medium from those of pre-hadrons in a color-neutral hot and dense fireball.

The authors acknowledge valuable discussions with E. L. Bratkovskaya, D. d'Enterria, Th. Falter, B. Jacak, C. M. Ko, and Z. W. Lin.

\section{References}

[1] J.D. Bjorken, Phys. Rev. D 27 (1983) 140.

[2] F. Karsch et al., Nucl. Phys. B 502 (2001) 321.

[3] Quark Matter '02, Nucl. Phys. A 715 (2003) 1.

[4] J. L. Nagle T. S. Ullrich, nucl-ex/0203007

[5] M. Gyulassy, M. Plumer, Phys. Lett. B 243 (1990) 432.

[6] X.-N. Wang, M. Gyulassy, Phys. Rev. Lett. 68 (1992) 1480.

[7] X. N. Wang, Phys. Rev. C 58 (1998) 2321.

[8] R. Baier et al., Ann. Rev. Nucl. Part. Sci. 50 (2000) 37.

[9] M. Gyulassy, I. Vitev, X. N. Wang B. Zhang, nucl-th/0302077, Contribution to the CERN yellow report on Hard Probes in Heavy Ion Collisions at the LHC. 
[10] S. S. Adler et al. [PHENIX], nucl-ex/0308006.

[11] J. Adams et al. [STAR], Phys. Rev. Lett. 91 (2003) 172302.

[12] I. Arsene et al. [BRAHMS], Phys. Rev. Lett. 91 (2003) 072305.

[13] S. S. Adler et al. [PHENIX], Phys. Rev. Lett. 91 (2003) 072303.

[14] J. Adams et al. [STAR], Phys. Rev. Lett. 91 (2003) 072304.

[15] X. N. Wang, Phys. Rev. C 61 (2000) 064910.

[16] Y. Zhang et al., Phys. Rev. C65 (2002) 034903.

[17] B. Z. Kopeliovich et al., Phys. Rev. Lett. 88 (2002) 232303.

[18] A. Accardi, hep-ph/0212148, to appear in: Quark Gluon Plasma 3, Editors: R.C. Hwa and X.-N. Wang, World Scientific, Singapore.

[19] I. Vitev, Phys. Lett. B562 (2003) 36.

[20] D. Kharzeev, E. Levin L. McLerran, Phys. Lett. B 561 (2003) 93.

[21] K. Gallmeister, C. Greiner, Z. Xu, Phys. Rev. C 67 (2003) 044905.

[22] W. Ehehalt, W. Cassing, Nucl. Phys. A 602 (1996) 449.

[23] J. Geiss, W. Cassing, C. Greiner, Nucl. Phys. A 644 (1998) 107.

[24] W. Cassing, E. L. Bratkovskaya, Phys. Rep. 308 (1999) 65.

[25] H. Pi, Comp. Phys. Commun. 71 (1992) 173.

[26] H.-U. Bengtsson, T. Sjöstrand, Comp. Phys. Commun. 46 (1987) 43.

[27] Z. W. Lin et al., Nucl. Phys. A 698 (2002) 375.

[28] H. Weber, E. L. Bratkovskaya, W. Cassing, H. Stöcker, Phys. Rev. C 67 (2003) 014904.

[29] V. Friese et al., [NA49], nucl-ex/0305017, to appear in J. Phys. G.

[30] E. L. Bratkovskaya, W. Cassing, H. Stöcker, Phys. Rev. C 67 (2003) 054905.

[31] T. Sjöstrand et al., Comp. Phys. Commun. 135 (2001) 238.

[32] K. Gallmeister, B. Kämpfer, O. P. Pavlenko, Nucl. Phys. A 688 (2001) 939; Phys. Rev. C 62 (2000) 057901.

[33] K. Gallmeister, B. Kämpfer, O. P. Pavlenko, Nucl. Phys. A 715 (2003) 705.

[34] S. S. Adler et al. [PHENIX], hep-ex/0304038

[35] B. Z. Kopeliovich, J. Nemchick, E. Predazzi, A. Hayashigaki, hep-ph/0311220. 
[36] S.A. Bass, M. Belkacem, M. Bleicher, M. Brandstetter, L. Bravina, C. Ernst, L. Gerland, M. Hofmann, S. Hofmann, J. Konopka, G. Mao, L. Neise, S. Soff, C. Spieles, H. Weber, L.A. Winckelmann, H. Stöcker, W. Greiner, Ch. Hartnack, J. Aichelin, and N. Amelin, Prog. Part. Nucl. Phys. 42 (1998) 279.

[37] M. Bleicher, E. Zabrodin, C. Spieles, S.A. Bass, C. Ernst, S. Soff, L. Bravina, M. Belkacem, H. Weber, H. Stöcker, and W. Greiner, J. Phys. G 25 (1999) 1859.

[38] W. Cassing, E. L. Bratkovskaya, A. Sibirtsev, Nucl. Phys. A 691 (2001) 753.

[39] J. Geiss, C. Greiner, E. L. Bratkovskaya, W. Cassing, U. Mosel, Phys. Lett. B 447 (1999) 31.

[40] W. Cassing, E. L. Bratkovskaya, Nucl. Phys. A 623 (1997) 570.

[41] W. Cassing, C. M. Ko, Phys. Lett. B 396 (1997) 39.

[42] E. L. Bratkovskaya, S. Soff. H. Stöcker, M. van Leuuwen, W. Cassing, nucl-th/0307098, Phys. Rev. Lett., in press.

[43] T. Falter, W. Cassing, K. Gallmeister, U. Mosel., nucl-th/0303011; nucl-th/0309057.

[44] A. Airapetian et al. [HERMES], Eur. Phys. J C21 (2001) 599; Phys. Lett. B 577 (2003) 37.

[45] W. Cassing, E. L. Bratkovskaya, O. Hansen, Nucl. Phys. A 707 (2002) 224.

[46] J. W. Cronin et al., Phys. Rev. D 11 (1975) 3105.

[47] D. Antreasyan et al., Phys. Rev. D 19 (1979) 764.

[48] G. G. Barnafoldi, G. Papp, P. Levai G. Fai, nucl-th/0307062.

[49] G. J. Alner et al. [UA5], Z. Phys. C 33 (1986) 1.

[50] B. B. Back et al. [PHOBOS], nucl-ex/0311009.

[51] B. Z. Kopeliovich, Phys. Rev. C 68 (2003) 044906.

[52] Y. Dokshitzer, V. Khoze, A. Mueller, S. Troyan, 'Basics of perturbative QCD', Editions Frontiers (1991).

[53] A. Drees, H. Feng, J. Jia, nucl-th/0310044.

[54] X.-N. Wang, nucl-th/0307036.

[55] P. F. Kolb et al., Phys. Rev. C 62 (2000) 054909; Nucl. Phys. A 696 (2001) 197.

[56] D. Molnar, M. Gyulassy, Nucl. Phys. A 698 (2002) 379; Nucl. Phys. A 697 (2002) 495.

[57] C. Adler et al. [STAR], Phys. Rev. Lett. 90 (2003) 082302.

[58] E. V. Shuryak, Phys. Rev. C 66 (2002) 027902.

[59] M. M. Aggarwal et al. [WA98], Eur. Phys. J. C 23 (2002) 225. 